\title{
New iterative methods for a common solution of fixed points for pseudo-contractive mappings and variational inequalities
}

\author{
Rabian Wangkeeree ${ }^{1}$ and Kamonrat Nammanee ${ }^{2^{*}}$
}

"Correspondence:

kamonrat.na@up.ac.th

${ }^{2}$ Department of Mathematics,

School of Science, University of

Phayao, Phayao, 56000, Thailand

Full list of author information is

available at the end of the article

\begin{abstract}
In this paper, we introduce three iterative methods for finding a common element of the set of fixed points for a continuous pseudo-contractive mapping and the solution set of a variational inequality problem governed by continuous monotone mappings. Strong convergence theorems for the proposed iterative methods are proved. Our results improve and extend some recent results in the literature.

MSC: $47 \mathrm{HO}$; 47H09; 47J25; 65J15
\end{abstract}

Keywords: pseudo-contractive mapping; monotone mapping; strong convergence theorem

\section{Introduction}

The theory of variational inequalities represents, in fact, a very natural generalization of the theory of boundary value problems and allows us to consider new problems arising from many fields of applied mathematics, such as mechanics, physics, engineering, the theory of convex programming, and the theory of control. While the variational theory of boundary value problems has its starting point in the method of orthogonal projection, the theory of variational inequalities has its starting point in the projection on a convex set.

Let $C$ be a nonempty closed and convex subset of a real Hilbert space $H$. The classical variational inequality problem is to find $u \in C$ such that $\langle v-u, A u\rangle \geq 0$ for all $v \in C$, where $A$ is a nonlinear mapping. The set of solutions of the variational inequality is denoted by $V I(C, A)$. The variational inequality problem has been extensively studied in the literature; see [1-9] and the references therein. In the context of the variational inequality problem, this implies that $u \in V I(C, A) \Leftrightarrow u=P_{C}(u-\lambda A u), \forall \lambda>0$, where $P_{C}$ is a metric projection of $H$ into $C$.

Let $A$ be a mapping from $C$ to $H$, then $A$ is called monotone if and only if for each $x, y \in C$,

$$
\langle x-y, A x-A x\rangle \geq 0 .
$$

An operator $A$ is said to be strongly positive on $H$ if there exists a constant $\bar{\gamma}>0$ such that

$$
\langle A x, x\rangle \geq \bar{\gamma}\|x\|^{2}, \quad \forall x \in H .
$$

2013 Wangkeeree and Nammanee; licensee Springer. This is an Open Access article distributed under the terms of the Creative Commons Attribution License (http://creativecommons.org/licenses/by/2.0), which permits unrestricted use, distribution, and reproduction in any medium, provided the original work is properly cited. 
A mapping $A$ of $C$ into itself is called $L$-Lipschitz continuous if there exits a positive number $L$ such that

$$
\|A x-A y\| \leq L\|x-y\|, \quad \forall x, y \in C .
$$

A mapping $A$ of $C$ into $H$ is called $\alpha$-inverse-strongly monotone if there exists a positive real number $\alpha$ such that

$$
\langle x-y, A x-A y\rangle \geq \alpha\|A x-A y\|^{2}
$$

for all $x, y \in C$; see [9-14]. If $A$ is an $\alpha$-inverse strongly monotone mapping of $C$ into $H$, then it is obvious that $A$ is $\frac{1}{\alpha}$-Lipschitz continuous, that is, $\|A x-A y\| \leq \frac{1}{\alpha}\|x-y\|$ for all $x, y \in C$. Clearly, the class of monotone mappings includes the class of $\alpha$-inverse strongly monotone mappings.

A mapping $A$ of $C$ into $H$ is called $\bar{\gamma}$-strongly monotone if there exists a positive real number $\bar{\gamma}$ such that

$$
\langle x-y, A x-A y\rangle \geq \bar{\gamma}\|x-y\|^{2}
$$

for all $x, y \in C$; see [15]. Clearly, the class of $\bar{\gamma}$-strongly monotone mappings includes the class of strongly positive mappings.

Recall that a mapping $T$ of $C$ into $H$ is called pseudo-contractive if for each $x, y \in C$, we have

$$
\langle T x-T y, x-y\rangle \leq\|x-y\|^{2} .
$$

$T$ is said to be a $k$-strict pseudo-contractive mapping if there exists a constant $0 \leq k \leq 1$ such that

$$
\langle x-y, T x-T y\rangle \leq\|x-y\|^{2}-k\|(I-T) x-(I-T) y\|^{2} \quad \text { for all } x, y \in D(T) .
$$

A mapping $T$ of $C$ into itself is called nonexpansive if $\|T x-T y\| \leq\|x-y\|$ for all $x, y \in C$. We denote by $F(T)$ the set of fixed points of $T$. Clearly, the class of pseudo-contractive mappings includes the class of nonexpansive and strict pseudo-contractive mappings.

For a sequence $\left\{\alpha_{n}\right\}$ of real numbers in $(0,1)$ and arbitrary $u \in C$, let the sequence $\left\{x_{n}\right\}$ in $C$ be iteratively defined by $x_{0} \in C$ and

$$
x_{n+1}:=\alpha_{n+1} u+\left(1-\alpha_{n+1}\right) T x_{n}, \quad n \geq 0,
$$

where $T$ is a nonexpansive mapping of $C$ into itself. Halpern [16] was first to study the convergence of algorithm (1.3) in the framework of Hilbert spaces. Lions [17] and Wittmann [18] improved the result of Halpern by proving strong convergence of $\left\{x_{n}\right\}$ to a fixed point of $T$ if the real sequence $\left\{\alpha_{n}\right\}$ satisfies certain conditions. Reich [19], Shioji and Takahashi [20], and Zegeye and Shahzad [21] extended the result of Wittmann [18] to the case of a Banach space. 
In 2000, Moudafi [22] introduced a viscosity approximation method and proved that if $H$ is a real Hilbert space, for given $x_{0} \in C$, the sequence $\left\{x_{n}\right\}$ generated by the algorithm

$$
x_{n+1}:=\alpha_{n} f\left(x_{n}\right)+\left(1-\alpha_{n}\right) T x_{n}, \quad n \geq 0,
$$

where $f: C \rightarrow C$ is a contraction mapping and $\left\{\alpha_{n}\right\} \subset(0,1)$ satisfies certain conditions, converges strongly to the unique solution $x^{*}$ in $C$ of the variational inequality

$$
\left\langle(I-f) x^{*}, x-x^{*}\right\rangle \geq 0, \quad x \in C .
$$

Moudafi [22] generalized Halpern's theorems in the direction of viscosity approximations. In [23], Zegeye et al. extended Moudafi's result to the case of Lipschitz pseudocontractive mappings in Banach spaces more general that Hilbert spaces.

In 2006, Marino and Xu [24] introduced the following general iterative method:

$$
x_{n+1}:=\alpha_{n} \gamma f\left(x_{n}\right)+\left(1-\alpha_{n} A\right) T x_{n}, \quad n \geq 0 .
$$

They proved that if the sequence $\left\{\alpha_{n}\right\}$ of parameters satisfies appropriate conditions, then the sequence $\left\{x_{n}\right\}$ generated by (1.6) converges strongly to the unique solution of the variational inequality

$$
\left\langle(A-\gamma f) x^{*}, x-x^{*}\right\rangle \geq 0, \quad x \in C,
$$

which is the optimality condition for the minimization problem

$$
\min _{x \in C} \frac{1}{2}\langle A x, x\rangle-h(x)
$$

where $h$ is a potential function for $\gamma f$ (i.e., $h^{\prime}(x)=\gamma f(x)$ for $x \in H$ ).

Recently, Zegeye and Shahzad [25] introduced an iterative method and proved that if $C$ is a nonempty subset of a real Hilbert space $H, T_{1}: C \rightarrow C$ is a pseudo-contractive mapping and $T_{2}: C \rightarrow H$ is a continuous monotone mapping such that $F:=F\left(T_{1}\right) \cap V I\left(C, T_{2}\right) \neq \emptyset$. For $\left\{r_{n}\right\} \subset(0, \infty)$ defined $T_{r_{n}}, F_{r_{n}}: H \rightarrow C$ by the following: for $x \in H$ and $\left\{r_{n}\right\} \subset(0, \infty)$, define

$$
\begin{aligned}
& T_{r_{n}} x:=\left\{z \in C:\left\langle y-z, T_{1} z\right\rangle-\frac{1}{r_{n}}\left\langle y-z,\left(1+r_{n}\right) z-x\right\rangle \leq 0, y \in C\right\}, \\
& F_{r_{n}} x:=\left\{z \in C:\left\langle y-z, T_{2} z\right\rangle+\frac{1}{r_{n}}\langle y-z, z-x\rangle \geq 0, y \in C\right\} .
\end{aligned}
$$

Then the sequence $\left\{x_{n}\right\}$ generated by $x_{1} \in C$ and

$$
x_{n+1}:=\alpha_{n} f\left(x_{n}\right)+\left(1-\alpha_{n}\right) T_{r_{n}} F_{r_{n}} x_{n}, \quad n \geq 1,
$$

where $f: C \rightarrow C$ is a contraction mapping and $\left\{\alpha_{n}\right\} \subset[0,1]$ and $\left\{r_{n}\right\}$ satisfy certain conditions, converges strongly to $z \in F$, where $z=P_{F} f(z)$.

In this paper, motivated and inspired by the method of Marino and $\mathrm{Xu}$ [24] and the work of Zegeye and Shahzad [25], we introduce a viscosity approximation method for finding a 
common fixed point of a set of fixed points of continuous pseudo-contractive mappings more general than nonexpansive mappings and a solution set of the variational inequality problem for continuous monotone mappings more general than $\alpha$-inverse strongly monotone mappings in a real Hilbert space. Our result extend and unify most of the results that have been proved for important classes of nonlinear operators.

Let $C$ be a nonempty closed and convex subset of a real Hilbert space $H$. Let $T_{1}, T_{2}: C \rightarrow$ $H$ be a continuous pseudo-contractive mapping and a continuous monotone mapping, respectively. For $x \in H$ and $\left\{r_{n}\right\} \subset(0, \infty)$, let $T_{r_{n}}, F_{r_{n}}: H \rightarrow C$ be defined by (1.8) and (1.9).

We consider the three iterative methods given as follows:

$$
\begin{aligned}
& x_{1} \in H, \\
& x_{n+1}:=\alpha_{n} \gamma f\left(x_{n}\right)+\left(I-\alpha_{n} A\right) T_{r_{n}} F_{r_{n}} x_{n}, \quad n \geq 1, \\
& y_{1} \in H, \\
& y_{n+1}:=\alpha_{n} \gamma f\left(T_{r_{n}} F_{r_{n}} y_{n}\right)+\left(I-\alpha_{n} A\right) T_{r_{n}} F_{r_{n}} y_{n}, \quad n \geq 1, \\
& z_{1} \in H, \\
& z_{n+1}:=T_{r_{n}} F_{r_{n}}\left(\alpha_{n} \gamma f\left(z_{n}\right)+\left(I-\alpha_{n} A\right) z_{n}\right), \quad n \geq 1,
\end{aligned}
$$

where $A$ is a $\bar{\gamma}$-strongly monotone and $L$-Lipschitzian continuous operator and $f: H \rightarrow H$ is a contraction mapping. We prove in Section 3 that if $\left\{\alpha_{n}\right\}$ and $\left\{r_{n}\right\}$ of parameters satisfy appropriate conditions, then the sequences $\left\{x_{n}\right\},\left\{y_{n}\right\}$ and $\left\{z_{n}\right\}$ converge strongly to $z=$ $P_{\mathfrak{F}}(I-A+\gamma f)(z)$.

\section{Preliminaries}

Let $C$ be a closed and convex subset of a real Hilbert space $H$. For every $x \in H$, there exists a unique nearest point in $C$, denoted by $P_{C} x$, such that

$$
\left\|x-P_{C} x\right\|=\|x-y\|, \quad \forall y \in C .
$$

$P_{C}$ is called the metric projection of $H$ onto $C$. We know that $P_{C}$ is a nonexpansive mapping of $H$ onto $C$. In connection with metric projection, we have the following lemma.

Lemma 2.1 Let H be a real Hilbert space. The following identity holds:

$$
\|x+y\|^{2} \leq\|x\|^{2}+2\langle y, x+y\rangle, \quad \forall x, y \in H .
$$

Lemma 2.2 Let $C$ be a nonempty closed convex subset of a Hilbert space H. Let $x \in H$ and $y \in C$. Then $y=P_{C} x$ if and only if

$$
\langle x-y, y-z\rangle \geq 0, \quad \forall z \in C
$$

Lemma 2.3 [26] Let $\left\{a_{n}\right\}$ be a sequence of nonnegative real numbers such that

$$
a_{n+1} \leq\left(1-\gamma_{n}\right) a_{n}+\sigma_{n}, \quad \forall n \geq 0,
$$


(i) $\left\{\gamma_{n}\right\} \subset[0,1], \sum_{n=1}^{\infty} \gamma_{n}=\infty$ and

(ii) $\limsup \sup _{n \rightarrow \infty} \frac{\sigma_{n}}{\gamma_{n}} \leq 0$ or $\sum_{n=1}^{\infty}\left|\sigma_{n}\right|<\infty$.

Then $a_{n} \rightarrow 0$ as $n \rightarrow \infty$.

Lemma 2.4 [27] Let $C$ be a nonempty closed and convex subset of a real Hilbert space $H$. Let $A: C \rightarrow H$ be a continuous monotone mapping. Then, for $r>0$ and $x \in H$, there exists $z \in C$ such that

$$
\langle y-z, A z\rangle+\frac{1}{r}\langle y-z, z-x\rangle \geq 0, \quad \forall y \in C .
$$

Moreover, by a similar argument as in the proof of Lemmas 2.8 and 2.9 in [28], Zegeye [27] obtained the following lemmas.

Lemma 2.5 [27] Let $C$ be a nonempty closed and convex subset of a real Hilbert space $H$. Let $A: C \rightarrow H$ be a continuous monotone mapping. For $r>0$ and $x \in H$, define a mapping $F_{r}: H \rightarrow C$ as follows:

$$
F_{r} x:=\left\{z \in C:\langle y-z, A z\rangle+\frac{1}{r}\langle y-z, z-x\rangle \geq 0, \forall y \in C\right\}
$$

for all $x \in H$. Then the following hold:

(1) $F_{r}$ is single-valued;

(2) $F_{r}$ is a firmly nonexpansive type mapping, i.e., for all $x, y \in H$,

$$
\left\|F_{r} x-F_{r} y\right\|^{2} \leq\left\langle F_{r} x-F_{r} y, x-y\right\rangle
$$

(3) $F\left(F_{r}\right)=V I(C, A)$;

(4) $\operatorname{VI}(C, A)$ is closed and convex.

In the sequel, we shall make use of the following lemmas.

Lemma 2.6 [27] Let $C$ be a nonempty closed and convex subset of a real Hilbert space $H$. Let $T: C \rightarrow H$ be a continuous pseudo-contractive mapping. Then, for $r>0$ and $x \in H$, there exists $z \in C$ such that

$$
\langle y-z, T z\rangle-\frac{1}{r}\langle y-z,(1+r) z-x\rangle \leq 0, \quad \forall y \in C .
$$

Lemma 2.7 [27] Let $C$ be a nonempty closed and convex subset of a real Hilbert space $H$. Let $T: C \rightarrow C$ be a continuous pseudo-contractive mapping. For $r>0$ and $x \in H$, define a mapping $T_{r}: H \rightarrow C$ as follows:

$$
T_{r} x:=\left\{z \in C:\langle y-z, T z\rangle+\frac{1}{r}\langle y-z,(1+r) z-x\rangle \leq 0, \forall y \in C\right\}
$$

for all $x \in H$. Then the following hold:

(1) $T_{r}$ is single-valued;

(2) $T_{r}$ is a firmly nonexpansive type mapping, i.e., for all $x, y \in H$,

$$
\left\|T_{r} x-T_{r} y\right\|^{2} \leq\left\langle T_{r} x-T_{r} y, x-y\right\rangle ;
$$


(3) $F\left(T_{r}\right)=F(T)$;

(4) $F(T)$ is closed and convex.

Lemma 2.8 [15] Let $0<\alpha<1$ and let $f$ be an $\alpha$-contraction of a real Hilbert space $H$ into itself, and let $A$ be a $\bar{\gamma}$-strongly monotone and L-Lipschitzian continuous operator of $H$ into itself with $\bar{\gamma}>0$ and $L>0$. Take $\mu, \gamma$ to be real numbers as follows:

$$
0<\mu<\frac{2 \bar{\gamma}}{L^{2}}, \quad 0<\gamma<\frac{\bar{\gamma}-\frac{L^{2} \mu}{2}}{\alpha} .
$$

If $\left\{\alpha_{n}\right\} \subseteq(0,1), \lim _{n \rightarrow \infty} \alpha_{n}=0$ and $\tau=\bar{\gamma}-\frac{L^{2} \mu}{2}$, then

$$
\left\|\left(I-\alpha_{n} A\right) x-\left(I-\alpha_{n} A\right) y\right\| \leq\left(1-\alpha_{n} \tau\right)\|x-y\|, \quad \forall x, y \in H .
$$

\section{Main results}

Now, we prove our main theorems.

Theorem 3.1 Let $H$ be a real Hilbertspace, $T_{1}: C \rightarrow C$ be a continuous pseudo-contractive mapping and $T_{2}: C \rightarrow H$ be a continuous monotone mapping such that $\mathfrak{F}:=F\left(T_{1}\right) \cap$ $\operatorname{VI}\left(C, T_{2}\right) \neq \emptyset$. Let $0<\alpha<1$ and let $f$ be an $\alpha$-contraction of $H$ into itself, and let $A$ be $a$ $\bar{\gamma}$-strongly monotone and L-Lipschitzian continuous operator of $C$ into $H$ with $\bar{\gamma}>0$ and $L>0$. Take $\mu, \gamma$ to be real numbers as follows:

$$
0<\mu<\frac{2 \bar{\gamma}}{L^{2}}, \quad 0<\gamma<\frac{\bar{\gamma}-\frac{L^{2} \mu}{2}}{\alpha} .
$$

For $x_{1} \in H$, let $\left\{x_{n}\right\}$ be a sequence generated by (1.11), where $\left\{\alpha_{n}\right\} \subset[0,1]$ and $\left\{r_{n}\right\} \subset(0, \infty)$ are such that $\lim _{n \rightarrow \infty} \alpha_{n}=0, \sum_{n=1}^{\infty} \alpha_{n}=\infty, \sum_{n=1}^{\infty}\left|\alpha_{n+1}-\alpha_{n}\right|<\infty, \liminf _{n \rightarrow \infty} r_{n}>0$ and $\sum_{n=1}^{\infty}\left|r_{n+1}-r_{n}\right|<\infty$. Then the sequence $\left\{x_{n}\right\}$ converges strongly to $z \in \mathfrak{F}$, where $z=P_{\mathfrak{F}}(I-$ $A+\gamma f)(z)$.

Proof Since $\alpha_{n} \rightarrow 0$ as $n \rightarrow \infty$, we may assume, without loss of generality, $\alpha_{n}<1$ for all $n$. For $Q=P_{\mathfrak{F}}$, it implies that $Q(I-A+\gamma f)$ is a contraction of $H$ into itself. Since $H$ is a real Hilbert space, there exists a unique element $z \in H$ such that $z=P_{\mathfrak{F}}(I-A+\gamma f)(z)$.

Let $v \in \mathfrak{F}$, and let $u_{n}:=T_{r_{n}} w_{n}$, where $w_{n}:=F_{r_{n}} x_{n}$. Then we have from Lemma (2.5) and (2.7) that

$$
\left\|u_{n}-v\right\|=\left\|T_{r_{n}} w_{n}-T_{r_{n}} v\right\| \leq\left\|w_{n}-v\right\|=\left\|F_{r_{n}} x_{n}-F_{r_{n}} v\right\| \leq\left\|x_{n}-v\right\| .
$$

Moreover, from (1.11) and (3.1), we get that

$$
\begin{aligned}
\left\|x_{n+1}-v\right\| & =\left\|\alpha_{n} \gamma f\left(x_{n}\right)+\left(I-\alpha_{n} A\right) u_{n}-v\right\| \\
& =\left\|\alpha_{n}\left(\gamma f\left(x_{n}\right)-A(v)\right)+\left(I-\alpha_{n} A\right) u_{n}-\left(I-\alpha_{n} A\right) v\right\| \\
& \leq\left(1-\alpha_{n} \tau\right)\left\|x_{n}-v\right\|+\alpha_{n} \gamma \alpha\left\|x_{n}-v\right\|+\alpha_{n}\|\gamma f(v)-A(v)\| \\
& \leq\left(1-\alpha_{n}(\tau-\gamma \alpha)\right)\left\|x_{n}-v\right\|+\alpha_{n}\|\gamma f(v)-A(v)\| \\
& =\left(1-\alpha_{n}(\tau-\gamma \alpha)\right)\left\|x_{n}-v\right\|+\alpha_{n}(\tau-\gamma \alpha) \frac{\|\gamma f(v)-A(v)\|}{\tau-\gamma \alpha} .
\end{aligned}
$$


It follows from induction that

$$
\left\|x_{n}-v\right\| \leq \max \left\{\left\|x_{1}-v\right\|, \frac{\|\gamma f(v)-A(v)\|}{\tau-\gamma \alpha}\right\}, \quad n \geq 1 .
$$

Thus $\left\{x_{n}\right\}$ is bounded, and hence so are $\left\{u_{n}\right\},\left\{w_{n}\right\}$ and $\left\{f\left(x_{n}\right)\right\}$. Next, to show that $\| x_{n+1}-$ $x_{n} \| \rightarrow 0$, we have

$$
\begin{aligned}
\left\|x_{n+1}-x_{n}\right\|= & \left\|\alpha_{n} \gamma f\left(x_{n}\right)+\left(I-\alpha_{n} A\right) u_{n}-\left(\alpha_{n-1} \gamma f\left(x_{n-1}\right)+\left(I-\alpha_{n-1} A\right) u_{n-1}\right)\right\| \\
= & \| \alpha_{n} \gamma f\left(x_{n}\right)-\alpha_{n} \gamma f\left(x_{n-1}\right)+\alpha_{n} \gamma f\left(x_{n-1}\right)-\alpha_{n-1} \gamma f\left(x_{n-1}\right) \\
& +\left(I-\alpha_{n} A\right) u_{n}-\left(I-\alpha_{n} A\right) u_{n-1}+\left(I-\alpha_{n} A\right) u_{n-1}-\left(I-\alpha_{n-1} A\right) u_{n-1} \| \\
\leq & \alpha_{n} \gamma \alpha\left\|x_{n}-x_{n-1}\right\|+\left|\alpha_{n}-\alpha_{n-1}\right| \gamma\left\|f\left(x_{n-1}\right)\right\| \\
& +\left(1-\alpha_{n} \tau\right)\left\|u_{n}-u_{n-1}\right\|+\left|\alpha_{n}-\alpha_{n-1}\right|\left\|A u_{n-1}\right\| \\
\leq & \alpha_{n} \gamma \alpha\left\|x_{n}-x_{n-1}\right\|+(1+\gamma)\left|\alpha_{n}-\alpha_{n-1}\right| K+\left(1-\alpha_{n} \tau\right)\left\|u_{n}-u_{n-1}\right\| \\
\leq & \alpha_{n} \gamma \alpha\left\|x_{n}-x_{n-1}\right\|+(1+\gamma)\left|\alpha_{n}-\alpha_{n-1}\right| K+\left(1-\alpha_{n} \tau\right)\left\|w_{n}-w_{n-1}\right\|,
\end{aligned}
$$

where $K=\sup \left\{\left\|f\left(x_{n}\right)\right\|+\left\|A u_{n}\right\|: n \in \mathbb{N}\right\}<\infty$.

Moreover, since $w_{n}=F_{r_{n}} x_{n}, w_{n+1}=F_{r_{n+1}} x_{n+1}$, we get that

$$
\begin{aligned}
& \left\langle y-w_{n}, T_{2} w_{n}\right\rangle+\frac{1}{r_{n}}\left\langle y-w_{n}, w_{n}-x_{n}\right\rangle \geq 0 \quad \text { for all } y \in C, \\
& \left\langle y-w_{n+1}, T_{2} w_{n+1}\right\rangle+\frac{1}{r_{n+1}}\left\langle y-w_{n+1}, w_{n+1}-x_{n+1}\right\rangle \geq 0 \quad \text { for all } y \in C .
\end{aligned}
$$

Putting $y=w_{n+1}$ in (3.5) and $y=w_{n}$ in (3.6), we get that

$$
\begin{aligned}
& \left\langle w_{n+1}-w_{n}, T_{2} w_{n}\right\rangle+\frac{1}{r_{n}}\left\langle w_{n+1}-w_{n}, w_{n}-x_{n}\right\rangle \geq 0, \\
& \left\langle w_{n}-w_{n+1}, T_{2} w_{n+1}\right\rangle+\frac{1}{r_{n+1}}\left\langle w_{n}-w_{n+1}, w_{n+1}-x_{n+1}\right\rangle \geq 0 .
\end{aligned}
$$

Adding (3.7) and (3.8), we have

$$
\left\langle w_{n+1}-w_{n}, T_{2} w_{n}-T_{2} w_{n+1}\right\rangle+\left\langle w_{n+1}-w_{n}, \frac{w_{n}-x_{n}}{r_{n}}-\frac{w_{n+1}-x_{n+1}}{r_{n+1}}\right\rangle \geq 0,
$$

which implies that

$$
-\left\langle w_{n+1}-w_{n}, T_{2} w_{n+1}-T_{2} w_{n}\right\rangle+\left\langle w_{n+1}-w_{n}, \frac{w_{n}-x_{n}}{r_{n}}-\frac{w_{n+1}-x_{n+1}}{r_{n+1}}\right\rangle \geq 0 .
$$

Now, using the fact that $T_{2}$ is monotone, we get that

$$
\left\langle w_{n+1}-w_{n}, \frac{w_{n}-x_{n}}{r_{n}}-\frac{w_{n+1}-x_{n+1}}{r_{n+1}}\right\rangle \geq 0,
$$

and hence

$$
\left\langle w_{n+1}-w_{n}, w_{n}-w_{n+1}+w_{n+1}-x_{n}-\frac{r_{n}}{r_{n+1}}\left(w_{n+1}-x_{n+1}\right)\right\rangle \geq 0 .
$$


Without loss of generality, let us assume that there exists a real number $b$ such that $r_{n}>b>0$ for all $n \in \mathbb{N}$. Then we have

$$
\begin{aligned}
\left\|w_{n+1}-w_{n}\right\|^{2} & \leq\left\langle w_{n+1}-w_{n}, x_{n+1}-x_{n}+\left(1-\frac{r_{n}}{r_{n+1}}\right)\left(w_{n+1}-x_{n+1}\right)\right\rangle \\
& \leq\left\|w_{n+1}-w_{n}\right\|\left\{\left\|x_{n+1}-x_{n}\right\|+\left|\left(1-\frac{r_{n}}{r_{n+1}}\right)\right|\left\|w_{n+1}-x_{n+1}\right\|\right\},
\end{aligned}
$$

and hence from (3.13) we obtain that

$$
\begin{aligned}
\left\|w_{n+1}-w_{n}\right\| & \leq\left\|x_{n+1}-x_{n}\right\|+\frac{1}{r_{n+1}}\left|r_{n+1}-r_{n}\right|\left\|w_{n+1}-x_{n+1}\right\| \\
& \leq\left\|x_{n+1}-x_{n}\right\|+\frac{1}{b}\left|r_{n+1}-r_{n}\right| L,
\end{aligned}
$$

where $L=\sup \left\{\left\|w_{n}-x_{n}\right\|: n \in \mathbb{N}\right\}<\infty$.

Furthermore, from (3.4) and (3.14) we have that

$$
\begin{aligned}
\left\|x_{n+1}-x_{n}\right\| \leq & \alpha_{n} \gamma \alpha\left\|x_{n}-x_{n-1}\right\|+(1+\gamma)\left|\alpha_{n}-\alpha_{n-1}\right| K \\
& +\left(1-\alpha_{n} \tau\right)\left(\left\|x_{n}-x_{n-1}\right\|+\frac{1}{b}\left|r_{n}-r_{n-1}\right| L\right) .
\end{aligned}
$$

Hence by Lemma 2.3, we have

$$
\left\|x_{n+1}-x_{n}\right\| \rightarrow 0 \quad \text { as } n \rightarrow \infty .
$$

Consequently, from (3.14) and (3.16), we have that

$$
\left\|w_{n+1}-w_{n}\right\| \rightarrow 0 \quad \text { as } n \rightarrow \infty
$$

Moreover, since $u_{n}=T_{r_{n}} w_{n}, u_{n+1}=T_{r_{n+1}} w_{n+1}$, we get that

$$
\left\langle y-u_{n}, T_{1} u_{n}\right\rangle-\frac{1}{r_{n}}\left\langle y-u_{n},\left(1+r_{n}\right) u_{n}-w_{n}\right\rangle \leq 0 \quad \text { for all } y \in C .
$$

and

$$
\left\langle y-u_{n+1}, T_{1} u_{n+1}\right\rangle-\frac{1}{r_{n+1}}\left\langle y-u_{n+1},\left(1+r_{n+1}\right) u_{n+1}-w_{n+1}\right\rangle \leq 0 \quad \text { for all } y \in C .
$$

Putting $y=u_{n+1}$ in (3.18) and $y=u_{n}$ in (3.19), we get that

$$
\left\langle u_{n+1}-u_{n}, T_{2} u_{n}\right\rangle-\frac{1}{r_{n}}\left\langle u_{n+1}-u_{n},\left(1+r_{n}\right) u_{n}-w_{n}\right\rangle \leq 0
$$

and

$$
\left\langle u_{n}-u_{n+1}, T_{2} u_{n+1}\right\rangle-\frac{1}{r_{n+1}}\left\langle u_{n}-u_{n+1},\left(1+r_{n+1}\right) u_{n+1}-w_{n+1}\right\rangle \leq 0 .
$$


Adding (3.20) and (3.21), we have

$$
\begin{aligned}
& \left\langle u_{n+1}-u_{n}, T_{2} u_{n}-T_{2} u_{n+1}\right\rangle \\
& \quad-\left\langle u_{n+1}-u_{n}, \frac{\left(1+r_{n}\right) u_{n}-w_{n}}{r_{n}}-\frac{\left(1+r_{n+1}\right) u_{n+1}-w_{n+1}}{r_{n+1}}\right\rangle \leq 0,
\end{aligned}
$$

which implies that

$$
\begin{aligned}
& \left\langle u_{n+1}-u_{n},\left(u_{n+1}-T_{2} u_{n+1}\right)-\left(u_{n}-T_{2} u_{n}\right)\right\rangle \\
& \quad-\left\langle u_{n+1}-u_{n}, \frac{u_{n}-w_{n}}{r_{n}}-\frac{u_{n+1}-w_{n+1}}{r_{n+1}}\right\rangle \leq 0 .
\end{aligned}
$$

Now, using the fact that $T_{1}$ is pseudo-contractive, we get that

$$
\left\langle u_{n+1}-u_{n}, \frac{u_{n}-w_{n}}{r_{n}}-\frac{u_{n+1}-w_{n+1}}{r_{n+1}}\right\rangle \geq 0,
$$

and hence

$$
\left\langle u_{n+1}-u_{n}, u_{n}-u_{n+1}+u_{n+1}-w_{n}-\frac{r_{n}}{r_{n+1}}\left(u_{n+1}-w_{n+1}\right)\right\rangle \geq 0 .
$$

Thus, using the method in (3.13) and (3.14), we have that

$$
\begin{aligned}
\left\|u_{n+1}-u_{n}\right\| & \leq\left\|x_{n+1}-x_{n}\right\|+\frac{1}{r_{n+1}}\left|r_{n+1}-r_{n}\right|\left\|u_{n+1}-w_{n+1}\right\| \\
& \leq\left\|x_{n+1}-x_{n}\right\|+\frac{1}{b}\left|r_{n+1}-r_{n}\right| L,
\end{aligned}
$$

where $L=\sup \left\{\left\|u_{n}-w_{n}\right\|: n \in \mathbb{N}\right\}<\infty$.

Therefore, from (3.16) and the property of $\left\{r_{n}\right\}$, we have that

$$
\left\|u_{n+1}-u_{n}\right\| \rightarrow 0 \quad \text { as } n \rightarrow \infty .
$$

Furthermore, since $x_{n}=\alpha_{n-1} \gamma f\left(x_{n-1}\right)+\left(I-\alpha_{n-1} A\right) u_{n-1}$, we have that

$$
\begin{aligned}
\left\|x_{n}-u_{n}\right\| & \leq\left\|x_{n}-u_{n-1}\right\|+\left\|u_{n-1}-u_{n}\right\| \\
& =\left\|\alpha_{n-1} \gamma f\left(x_{n-1}\right)+\left(I-\alpha_{n-1} A\right) u_{n-1}-u_{n-1}\right\|+\left\|u_{n-1}-u_{n}\right\| \\
& =\alpha_{n-1}\left\|\gamma f\left(x_{n-1}\right)-A u_{n-1}\right\|+\left\|u_{n-1}-u_{n}\right\| .
\end{aligned}
$$

From $\alpha_{n} \rightarrow 0$, we have $\left\|x_{n}-u_{n}\right\| \rightarrow 0$.

Now, for $v \in \mathfrak{F}$, using Lemma 2.5, we get that

$$
\begin{aligned}
\left\|w_{n}-v\right\|^{2} & =\left\|F_{r_{n}} x_{n}-F_{r_{n}} v\right\|^{2} \\
& \leq\left\langle F_{r_{n}} x_{n}-F_{r_{n}} v, x_{n}-v\right\rangle \\
& =\left\langle w_{n}-v, x_{n}-v\right\rangle \\
& =\frac{1}{2}\left(\left\|w_{n}-v\right\|^{2}+\left\|x_{n}-v\right\|^{2}-\left\|x_{n}-w_{n}\right\|^{2}\right),
\end{aligned}
$$


and hence

$$
\left\|w_{n}-v\right\|^{2} \leq\left\|x_{n}-v\right\|^{2}-\left\|x_{n}-w_{n}\right\|^{2} .
$$

Therefore, we have

$$
\begin{aligned}
\left\|x_{n+1}-v\right\|^{2}= & \left\|\alpha_{n} \gamma f\left(x_{n}\right)+\left(I-\alpha_{n} A\right) u_{n}-v\right\|^{2} \\
= & \left\|\alpha_{n}\left(\gamma f\left(x_{n}\right)-A v\right)+\left(I-\alpha_{n} A\right)\left(u_{n}-v\right)\right\|^{2} \\
\leq & \left(1-\alpha_{n} \tau\right)^{2}\left\|u_{n}-v\right\|^{2}+2 \alpha_{n}\left\langle\gamma f\left(x_{n}\right)-A v, x_{n+1}-v\right\rangle \\
\leq & \left(1-\alpha_{n} \tau\right)^{2}\left\|w_{n}-v\right\|^{2}+2 \alpha_{n}\left\langle\gamma f\left(x_{n}\right)-\gamma f(v)+\gamma f(v)-A v, x_{n+1}-v\right\rangle \\
\leq & \left(1-\alpha_{n} \tau\right)^{2}\left\|w_{n}-v\right\|^{2}+2 \alpha_{n} \gamma\left\langle f\left(x_{n}\right)-f(v), x_{n+1}-v\right\rangle \\
& +2 \alpha_{n}\left(\gamma f(v)-A v, x_{n+1}-v\right\rangle \\
\leq & \left(1-\alpha_{n} \tau\right)^{2}\left\|w_{n}-v\right\|^{2}+2 \alpha_{n} \gamma \alpha\left\|x_{n}-v\right\|\left\|x_{n+1}-v\right\| \\
& +2 \alpha_{n}\|\gamma f(v)-A v\|\left\|x_{n+1}-v\right\| \\
\leq & \left(1-\alpha_{n} \tau\right)^{2}\left(\left\|x_{n}-v\right\|^{2}-\left\|x_{n}-w_{n}\right\|^{2}\right)+2 \alpha_{n} \gamma \alpha\left\|x_{n}-v\right\|\left\|x_{n+1}-v\right\| \\
& +2 \alpha_{n}\|\gamma f(v)-A v\|\left\|x_{n+1}-v\right\| \\
= & \left(1-2 \alpha_{n} \tau+\left(\alpha_{n} \tau\right)^{2}\right)\left\|x_{n}-v\right\|^{2}-\left(1-\alpha_{n} \tau\right)^{2}\left\|x_{n}-w_{n}\right\|^{2} \\
& +2 \alpha_{n} \gamma \alpha\left\|x_{n}-v\right\|\left\|x_{n+1}-v\right\|+2 \alpha_{n}\|\gamma f(v)-A v\|\left\|x_{n+1}-v\right\| .
\end{aligned}
$$

Hence

$$
\begin{aligned}
(1- & \left.\alpha_{n} \tau\right)^{2}\left\|x_{n}-w_{n}\right\|^{2} \\
\leq & \left\|x_{n}-v\right\|^{2}-\left\|x_{n+1}-v\right\|^{2}+\alpha_{n} \bar{\gamma}^{2}\left\|x_{n}-v\right\|^{2} \\
& +2 \alpha_{n} \gamma \alpha\left\|x_{n}-v\right\|\left\|x_{n+1}-v\right\|+2 \alpha_{n}\|\gamma f(v)-A v\|\left\|x_{n+1}-v\right\| \\
\leq & \left\|x_{n}-x_{n+1}\right\|\left\{\left\|x_{n}-v\right\|+\left\|x_{n+1}-v\right\|\right\}+\alpha_{n} \tau^{2}\left\|x_{n}-v\right\|^{2} \\
& +2 \alpha_{n} \gamma \alpha\left\|x_{n}-v\right\|\left\|x_{n+1}-v\right\|+2 \alpha_{n}\|\gamma f(v)-A v\|\left\|x_{n+1}-v\right\| .
\end{aligned}
$$

So, we have

$$
\left\|x_{n}-w_{n}\right\| \rightarrow 0 \quad \text { as } n \rightarrow \infty .
$$

Since $\left\|u_{n}-w_{n}\right\| \leq\left\|u_{n}-x_{n}\right\|+\left\|x_{n}-w_{n}\right\|$, it follows that

$$
\left\|u_{n}-w_{n}\right\| \rightarrow 0 \quad \text { as } n \rightarrow \infty .
$$

Next, we show that

$$
\limsup _{n \rightarrow \infty}\left\langle(A-\gamma f) z, z-x_{n}\right\rangle \leq 0
$$

where $z=P_{\mathfrak{F}}(I-A+\gamma f)(z)$. 
To show this equality, we choose a subsequence $\left\{x_{n_{i}}\right\}$ of $\left\{x_{n}\right\}$ such that

$$
\limsup _{n \rightarrow \infty}\left\langle(A-\gamma f) z, z-x_{n_{i}}\right\rangle=\limsup _{n \rightarrow \infty}\left\langle(A-\gamma f) z, z-x_{n}\right\rangle .
$$

Since $\left\{x_{n_{i}}\right\}$ is bounded, there exists a subsequence $\left\{x_{n_{i j}}\right\}$ of $\left\{x_{n_{i}}\right\}$ and $w \in H$ such that $x_{n_{i_{j}}} \rightarrow w$. Without loss of generality, we may assume that $x_{n_{i}} \rightarrow w$. Since $\left\{x_{n_{i}}\right\} \subset H$ and $H$ is closed and convex, we get that $w \in H$. Moreover, since $x_{n}-w_{n} \rightarrow 0$ as $n \rightarrow \infty$, we have that $w_{n_{i}} \rightarrow w$.

Now, we show that $w \in \mathfrak{F}$. Note that from the definition of $w_{n}$, we have

$$
\left\langle y-w_{n_{i}}, T_{2} w_{n_{i}}\right\rangle+\left\langle y-w_{n_{i}}, \frac{w_{n_{i}}-x_{n_{i}}}{r_{n_{i}}}\right\rangle \geq 0 \quad \text { for all } y \in C .
$$

Put $z_{t}=t v+(1-t) w$ for all $t \in(0,1]$ and $v \in H$. Consequently, we get that $z_{t} \in H$. From (3.34) it follows that

$$
\begin{aligned}
\left\langle z_{t}-w_{n_{i}}, T_{2} z_{t}\right\rangle & \geq\left\langle z_{t}-w_{n_{i}}, T_{2} z_{t}\right\rangle-\left\langle z_{t}-w_{n_{i}}, T_{2} w_{n_{i}}\right\rangle-\left\langle z_{t}-w_{n_{i}}, \frac{w_{n_{i}}-x_{n_{i}}}{r_{n_{i}}}\right\rangle \\
& =\left\langle z_{t}-w_{n_{i}}, T_{2} z_{t}-T_{2} w_{n_{i}}\right\rangle-\left\langle z_{t}-w_{n_{i}}, \frac{w_{n_{i}}-x_{n_{i}}}{r_{n_{i}}}\right\rangle .
\end{aligned}
$$

From the fact that $w_{n_{i}}-x_{n_{i}} \rightarrow 0$ as $n \rightarrow \infty$, we obtain that $\frac{w_{n_{i}}-x_{n_{i}}}{r_{n_{i}}} \rightarrow 0$ as $n \rightarrow \infty$.

Since $T_{2}$ is monotone, we have that $\left\langle z_{t}-w_{n_{i}}, T_{2} z_{t}-T_{2} w_{n_{i}}\right\rangle \geq 0$. Thus, it follows that

$$
0 \leq \lim _{i \rightarrow \infty}\left\langle z_{t}-w_{n_{i}}, T_{2} z_{t}\right\rangle=\left\langle z_{t}-w, T_{2} z_{t}\right\rangle
$$

and hence

$$
\left\langle z-w, T_{2} z_{t}\right\rangle \geq 0 \quad \text { for all } z \in C \text {. }
$$

Letting $t \rightarrow 0$ and using the fact that $T_{2}$ is continuous, we obtain that

$$
\left\langle z-w, T_{2} w\right\rangle \geq 0 \quad \text { for all } v \in C .
$$

This implies that $w \in \operatorname{VI}\left(C, T_{2}\right)$.

Furthermore, from the definition of $u_{n_{i}}$ we have that

$$
\left\langle y-u_{n_{i}}, T_{2} u_{n_{i}}\right\rangle-\frac{1}{r_{n_{i}}}\left\langle y-u_{n_{i}},\left(r_{n_{i}}+1\right) u_{n_{i}}-x_{n_{i}}\right\rangle \leq 0 \quad \text { for all } y \in C \text {. }
$$

Put $z_{t}=t v+(1-t) w$ for all $t \in(0,1]$ and $v \in H$. Consequently, we get that $z_{t} \in H$. From (3.33) and pseudo-contractivity of $T_{1}$, it follows that

$$
\begin{aligned}
\left\langle u_{n_{i}}-z_{t}, T_{1} z_{t}\right\rangle & \geq\left\langle u_{n_{i}}-z_{t}, T_{1} z_{t}\right\rangle+\left\langle z_{t}-u_{n_{i}}, T_{1} u_{n_{i}}\right\rangle-\frac{1}{r_{n_{i}}}\left\langle z_{t}-u_{n_{i}},\left(r_{n_{i}}+1\right) u_{n_{i}}-w_{n_{i}}\right\rangle \\
& =-\left\langle z_{t}-u_{n_{i}}, T_{1} z_{t}-T_{1} u_{n_{i}}\right\rangle-\frac{1}{r_{n_{i}}}\left\langle z_{t}-u_{n_{i}}, u_{n_{i}}-w_{n_{i}}\right\rangle-\left\langle z_{t}-u_{n_{i}}, u_{n_{i}}\right\rangle
\end{aligned}
$$




$$
\begin{aligned}
& \geq-\left\|z_{t}-u_{n_{i}}\right\|^{2}-\frac{1}{r_{n_{i}}}\left\langle z_{t}-u_{n_{i}}, u_{n_{i}}-w_{n_{i}}\right\rangle-\left\langle z_{t}-u_{n_{i}}, u_{n_{i}}\right\rangle \\
& =-\left\langle z_{t}-u_{n_{i}}, z_{t}\right\rangle-\left\langle z_{t}-u_{n_{i}}, \frac{u_{n_{i}}-w_{n_{i}}}{r_{n_{i}}}\right\rangle .
\end{aligned}
$$

Then, since $w_{n}-x_{n} \rightarrow 0$ as $n \rightarrow \infty$, we obtain that $\frac{w_{n_{i}}-x_{n_{i}}}{r_{n_{i}}} \rightarrow 0$ as $i \rightarrow \infty$. Thus, as $i \rightarrow \infty$, it follows that

$$
\left\langle w-z_{t}, T_{1} z_{t}\right\rangle \geq\left\langle w-z_{t}, z_{t}\right\rangle
$$

and hence

$$
-\left\langle v-w, T_{1} z_{t}\right\rangle \geq-\left\langle v-w, z_{t}\right\rangle \quad \text { for all } v \in C
$$

Letting $t \rightarrow 0$ and using the fact that $T_{1}$ is continuous, we obtain that

$$
-\left\langle v-w, T_{1} w\right\rangle \geq-\langle v-w, w\rangle \quad \text { for all } v \in C .
$$

Now, let $v=T_{1} w$. Then we obtain that $w=T_{1} w$ and hence $w \in F\left(T_{1}\right)$.

Therefore, $w \in F\left(T_{1}\right) \cap V I\left(C, T_{2}\right)$ and since $z=P_{\mathfrak{F}}(I-A+\gamma f)(z)$, by Lemma 2.2, implies that

$$
\begin{aligned}
\limsup _{n \rightarrow \infty}\left\langle(\gamma f-A)(z), x_{n}-z\right\rangle & =\limsup _{i \rightarrow \infty}\left\langle(I-A+\gamma f)(z), x_{n_{i}}-z\right\rangle \\
& =\langle(\gamma f-A)(z), w-z\rangle \leq 0 .
\end{aligned}
$$

Now, we show that $x_{n} \rightarrow z$ as $n \rightarrow \infty$. From $x_{n+1}-z=\alpha_{n}\left(\gamma f\left(x_{n}\right)-A z\right)+\left(I-\alpha_{n} A\right)\left(u_{n}-z\right)$, we have that

$$
\begin{aligned}
\left\|x_{n+1}-z\right\|^{2}= & \left\|\alpha_{n}\left(\gamma f\left(x_{n}\right)-A z\right)+\left(I-\alpha_{n} A\right)\left(u_{n}-z\right)\right\|^{2} \\
\leq & \left(1-\alpha_{n} \tau\right)^{2}\left\|u_{n}-z\right\|^{2}+2 \alpha_{n}\left\langle\gamma f\left(x_{n}\right)-A z, x_{n+1}-z\right\rangle \\
\leq & \left(1-\alpha_{n} \tau\right)^{2}\left\|x_{n}-z\right\|^{2} \\
& +2 \alpha_{n}\left\langle\gamma f\left(x_{n}\right)-\gamma f(z)+\gamma f(z)-A z, x_{n+1}-z\right\rangle \\
\leq & \left(1-\alpha_{n} \tau\right)^{2}\left\|x_{n}-z\right\|^{2}+2 \alpha_{n} \gamma\left\langle f\left(x_{n}\right)-f(z), x_{n+1}-z\right\rangle \\
& +2 \alpha_{n}\left\langle\gamma f(z)-A z, x_{n+1}-z\right\rangle \\
\leq & \left(1-\alpha_{n} \tau\right)^{2}\left\|x_{n}-z\right\|^{2}+2 \alpha_{n} \gamma \alpha\left\|x_{n}-z\right\|\left\|x_{n+1}-z\right\| \\
& +2 \alpha_{n}\left\langle\gamma f(z)-A z, x_{n+1}-z\right\rangle \\
\leq & \left(1-\alpha_{n} \tau\right)^{2}\left\|x_{n}-z\right\|^{2}+\alpha_{n} \gamma \alpha\left\{\left\|x_{n}-z\right\|^{2}+\left\|x_{n+1}-z\right\|^{2}\right\} \\
& +2 \alpha_{n}\left\langle\gamma f(z)-A z, x_{n+1}-z\right\rangle \\
= & \left(\left(1-\alpha_{n} \tau\right)^{2}+\alpha_{n} \gamma \alpha\right)\left\|x_{n}-z\right\|^{2}+\alpha_{n} \gamma \alpha\left\|x_{n+1}-z\right\|^{2} \\
& +2 \alpha_{n}\left\langle\gamma f(z)-A z, x_{n+1}-z\right\rangle .
\end{aligned}
$$


This implies that

$$
\begin{aligned}
\left\|x_{n+1}-z\right\|^{2} \leq & \frac{1-2 \alpha_{n} \tau+\left(\alpha_{n} \tau\right)^{2}+\alpha_{n} \gamma \alpha}{1-\alpha_{n} \gamma \alpha}\left\|x_{n}-z\right\|^{2} \\
& +\frac{2 \alpha_{n}}{1-\alpha_{n} \gamma \alpha}\left\langle\gamma f(z)-A z, x_{n+1}-z\right\rangle \\
= & {\left[1-\frac{2(\tau-\gamma \alpha) \alpha_{n}}{1-\alpha_{n} \gamma \alpha}\right]\left\|x_{n}-z\right\|^{2}+\frac{\left(\alpha_{n} \tau\right)^{2}}{1-\alpha_{n} \gamma \alpha}\left\|x_{n}-z\right\|^{2} } \\
& +\frac{2 \alpha_{n}}{1-\alpha_{n} \gamma \alpha}\left\langle\gamma f(z)-A z, x_{n+1}-z\right\rangle \\
\leq & {\left[1-\frac{2(\tau-\gamma \alpha) \alpha_{n}}{1-\alpha_{n} \gamma \alpha}\right]\left\|x_{n}-z\right\|^{2} } \\
& +\frac{2(\tau-\gamma \alpha) \alpha_{n}}{1-\alpha_{n} \gamma \alpha}\left\{\frac{\left(\alpha_{n} \tau\right) M}{2(\bar{\gamma}-\gamma \alpha)}+\frac{1}{\tau-\gamma \alpha}\left\langle\gamma f(z)-A z, x_{n+1}-z\right\rangle\right\} \\
= & \left(1-\delta_{n}\right)\left\|x_{n}-z\right\|^{2}+\delta_{n} \beta_{n},
\end{aligned}
$$

where $M=\sup \left\{\left\|x_{n}-z\right\|^{2}: n \in \mathbb{N}\right\}, \delta_{n}=\frac{2(\tau-\gamma \alpha) \alpha_{n}}{1-\alpha_{n} \gamma \alpha}$ and $\beta_{n}=\frac{\left(\alpha_{n} \tau\right) M}{2(\tau-\gamma \alpha)}+\frac{1}{\tau-\gamma \alpha}\left\langle\gamma f(z)-A z, x_{n+1}-\right.$ $z\rangle$. We put $\xi_{n}=\delta_{n} \beta_{n}$. It is easy to see that $\delta_{n} \rightarrow 0, \sum_{n=1}^{\infty} \delta_{n}=\infty$ and $\lim _{\sup } \operatorname{sum}_{n \rightarrow \infty} \frac{\xi}{n}_{n} \leq 0$ by (3.42). Hence, by Lemma 2.3, the sequence $\left\{x_{n}\right\}$ converges strongly to $z$. This completes the proof.

Theorem 3.2 Let $H$ be a real Hilbert space, $T_{1}: C \rightarrow H$ be a continuous pseudocontractive mapping and $T_{2}: C \rightarrow H$ be a continuous monotone mapping such that $\mathfrak{F}:=$ $F\left(T_{1}\right) \cap V I\left(C, T_{2}\right) \neq \emptyset$. Let $0<\alpha<1$ and let $f$ be an $\alpha$-contraction of $H$ into itself, and let $A$ be a $\bar{\gamma}$-strongly monotone and L-Lipschitzian continuous operator of $H$ into itself $H$ with $\bar{\gamma}>0$ and $L>0$. Take $\mu, \gamma$ to be real numbers as follows:

$$
0<\mu<\frac{2 \bar{\gamma}}{L^{2}}, \quad 0<\gamma<\frac{\bar{\gamma}-\frac{L^{2} \mu}{2}}{\alpha} .
$$

For $y_{1} \in H$, let $\left\{y_{n}\right\}$ be a sequence generated by (1.12), where $\left\{\alpha_{n}\right\} \subset[0,1]$ and $\left\{r_{n}\right\} \subset(0, \infty)$ are such that $\lim _{n \rightarrow \infty} \alpha_{n}=0, \sum_{n=1}^{\infty} \alpha_{n}=\infty, \sum_{n=1}^{\infty}\left|\alpha_{n+1}-\alpha_{n}\right|<\infty, \liminf _{n \rightarrow \infty} r_{n}>0$ and $\sum_{n=1}^{\infty}\left|r_{n+1}-r_{n}\right|<\infty$. The sequence $\left\{y_{n}\right\}$ converges strongly to $z \in \mathfrak{F}$, where $z=P_{\mathfrak{F}}(I-A+$ $\gamma f)(z)$.

Proof Let $\left\{x_{n}\right\}$ be the sequence given by $x_{1}=y_{1}$ and

$$
x_{n+1}=\alpha_{n} \gamma f\left(x_{n}\right)+\left(I-\alpha_{n} A\right) T_{r_{n}} F_{r_{n}} x_{n}, \quad \forall n \geq 1 .
$$

From Theorem 3.1, $x_{n} \rightarrow z$. We claim that $y_{n} \rightarrow z$. Indeed, we estimate

$$
\begin{aligned}
\left\|x_{n+1}-y_{n+1}\right\| \leq & \left\|\alpha_{n} \gamma f\left(x_{n}\right)-\alpha_{n} \gamma f\left(T_{r_{n}} F_{r_{n}} y_{n}\right)\right\| \\
& +\left\|\left(I-\alpha_{n} A\right) T_{r_{n}} F_{r_{n}} x_{n}-\left(I-\alpha_{n} A\right) T_{r_{n}} F_{r_{n}} y_{n}\right\| \\
\leq & \alpha_{n} \gamma \alpha\left\|T_{r_{n}} F_{r_{n}} y_{n}-x_{n}\right\|+\left(1-\alpha_{n} \tau\right)\left\|x_{n}-y_{n}\right\| \\
\leq & \alpha_{n} \gamma \alpha\left\|T_{r_{n}} F_{r_{n}} y_{n}-T_{r_{n}} F_{r_{n}} z\right\|+\alpha_{n} \gamma \alpha\left\|T_{r_{n}} F_{r_{n}} z-x_{n}\right\|+\left(1-\alpha_{n} \tau\right)\left\|x_{n}-y_{n}\right\|
\end{aligned}
$$




$$
\begin{aligned}
& \leq \alpha_{n} \gamma \alpha\left\|y_{n}-z\right\|+\alpha_{n} \gamma \alpha\left\|z-x_{n}\right\|+\left(1-\alpha_{n} \tau\right)\left\|x_{n}-y_{n}\right\| \\
& \leq \alpha_{n} \gamma \alpha\left\|y_{n}-x_{n}\right\|+\alpha_{n} \gamma \alpha\left\|x_{n}-z\right\|+\alpha_{n} \gamma \alpha\left\|z-x_{n}\right\|+\left(1-\alpha_{n} \tau\right)\left\|x_{n}-y_{n}\right\| \\
& =\left(1-\alpha_{n}(\tau-\gamma \alpha)\right)\left\|x_{n}-y_{n}\right\|+\alpha_{n}(\tau-\gamma \alpha) \frac{2 \gamma \alpha}{\tau-\gamma \alpha}\left\|x_{n}-z\right\|
\end{aligned}
$$

It follows from $\sum_{n=1}^{\infty} \alpha_{n}=\infty, \lim _{n \rightarrow \infty}\left\|x_{n}-z\right\|=0$ and Lemma 2.3 that $\left\|x_{n}-y_{n}\right\| \rightarrow 0$. Consequently, $y_{n} \rightarrow z$ as required.

Theorem 3.3 Let $H$ be a real Hilbert space, $T_{1}: C \rightarrow H$ be a continuous pseudocontractive mapping and $T_{2}: C \rightarrow H$ be a continuous monotone mapping such that $\mathfrak{F}:=$ $F\left(T_{1}\right) \cap V I\left(C, T_{2}\right) \neq \emptyset$. Let $0<\alpha<1$ and let $f$ be an $\alpha$-contraction of $H$ into itself, and let $A$ be a $\bar{\gamma}$-strongly monotone and L-Lipschitzian continuous operator of $H$ into itself $H$ with $\bar{\gamma}>0$ and $L>0$. Take $\mu, \gamma$ to be real numbers as follows:

$$
0<\mu<\frac{2 \bar{\gamma}}{L^{2}}, \quad 0<\gamma<\frac{\bar{\gamma}-\frac{L^{2} \mu}{2}}{\alpha} .
$$

For $z_{1} \in H$, let $\left\{z_{n}\right\}$ be a sequence generated by (1.13), where $\left\{\alpha_{n}\right\} \subset[0,1]$ and $\left\{r_{n}\right\} \subset(0, \infty)$ are such that $\lim _{n \rightarrow \infty} \alpha_{n}=0, \sum_{n=1}^{\infty} \alpha_{n}=\infty, \sum_{n=1}^{\infty}\left|\alpha_{n+1}-\alpha_{n}\right|<\infty, \liminf _{n \rightarrow \infty} r_{n}>0$ and $\sum_{n=1}^{\infty}\left|r_{n+1}-r_{n}\right|<\infty$. The sequence $\left\{z_{n}\right\}$ converges strongly to $z \in \mathfrak{F}$, where $z=P_{\mathfrak{F}}(I-A+$ $\gamma f)(z)$.

Proof Define the sequences $\left\{y_{n}\right\}$ and $\left\{\beta_{n}\right\}$ by

$$
y_{n}=\alpha_{n} \gamma f\left(z_{n}\right)+\left(I-\alpha_{n} A\right) z_{n} \quad \text { and } \quad \beta_{n}=\alpha_{n+1}, \quad \forall n \geq 1 .
$$

Taking $p \in \mathfrak{F}$, we have

$$
\begin{aligned}
\left\|z_{n+1}-p\right\| & =\left\|T_{r_{n}} F_{r_{n}} y_{n}-T_{r_{n}} F_{r_{n}} p\right\| \leq\left\|y_{n}-p\right\| \\
& =\left\|\alpha_{n} \gamma f\left(z_{n}\right)+\left(I-\alpha_{n} A\right) z_{n}-\left(I-\alpha_{n} A\right) p-\alpha_{n} A(p)\right\| \\
& \leq\left(1-\alpha_{n} \tau\right)\left\|z_{n}-p\right\|+\alpha_{n}\left\|\gamma f\left(z_{n}\right)-A(p)\right\| \\
& =\left(1-\alpha_{n} \tau\right)\left\|z_{n}-p\right\|+\alpha_{n} \tau \frac{\left\|\gamma f\left(z_{n}\right)-A(p)\right\|}{\tau} .
\end{aligned}
$$

It follows from induction that

$$
\left\|z_{n+1}-p\right\| \leq \max \left\{\left\|z_{1}-p\right\|, \frac{\left\|\gamma f\left(z_{1}\right)-A(p)\right\|}{\tau}\right\}, \quad n \geq 1 .
$$

Thus both $\left\{z_{n}\right\}$ and $\left\{y_{n}\right\}$ are bounded. We observe that

$$
y_{n+1}=\alpha_{n+1} \gamma f\left(z_{n+1}\right)+\left(I-\alpha_{n+1} A\right) z_{n+1}=\beta_{n} \gamma f\left(T_{r_{n}} F_{r_{n}} y_{n}\right)+\left(I-\beta_{n} A\right) T_{r_{n}} F_{r_{n}} y_{n} .
$$

Thus Theorem 3.2 implies that $\left\{y_{n}\right\}$ converges to some point $z$. In this case, we also have

$$
\left\|z_{n}-z\right\| \leq\left\|z_{n}-y_{n}\right\|+\left\|y_{n}-z\right\|=\alpha_{n}\left\|\gamma f\left(z_{n}\right)-A\left(z_{n}\right)\right\|+\left\|y_{n}-z\right\| \rightarrow 0 .
$$


Hence the sequence $\left\{z_{n}\right\}$ converges to some point $z$. This completes the proof.

Setting $\gamma=1, A \equiv I$, where $I$ is the identity mapping in Theorem 3.1, we have the following result.

Corollary 3.4 Let $H$ be a real Hilbert space, $T_{1}: C \rightarrow C$ be a continuous pseudocontractive mapping and $T_{2}: C \rightarrow H$ be a continuous monotone mapping such that $\mathfrak{F}:=$ $F\left(T_{1}\right) \cap V I\left(C, T_{2}\right) \neq \emptyset$. Let $f$ be a contraction of $H$ into itself and let $\left\{x_{n}\right\}$ be a sequence generated by $x_{1} \in H$ and

$$
x_{n+1}=\alpha_{n} f\left(x_{n}\right)+\left(1-\alpha_{n}\right) F_{r_{n}} T_{r_{n}} x_{n},
$$

where $\left\{\alpha_{n}\right\} \subset[0,1]$ and $\left\{r_{n}\right\} \subset(0, \infty)$ are such that $\lim _{n \rightarrow \infty} \alpha_{n}=0, \sum_{n=0}^{\infty} \alpha_{n}=\infty$, $\sum_{n=0}^{\infty}\left|\alpha_{n+1}-\alpha_{n}\right|<\infty, \liminf _{n \rightarrow \infty} r_{n}>0$ and $\sum_{n=0}^{\infty}\left|r_{n+1}-r_{n}\right|<\infty$. The sequence $\left\{x_{n}\right\}$ converges strongly to $z \in \mathfrak{F}$, where $z=P_{\mathfrak{F}} f(z)$.

In Theorem 3.1, $\gamma=1, A \equiv I, f:=u \in H$ is a constant mapping, then we get $z=P_{\mathfrak{F}}(u)$. In fact, we have the following corollary.

Corollary 3.5 Let $H$ be a real Hilbert space, $T_{1}: C \rightarrow C$ be a continuous pseudocontractive mapping and $T_{2}: C \rightarrow H$ be a continuous monotone mapping such that $\mathfrak{F}:=$ $F\left(T_{1}\right) \cap V I\left(C, T_{2}\right) \neq \emptyset$. Let $\left\{x_{n}\right\}$ be a sequence generated by $x_{1}, u \in H$ and

$$
x_{n+1}=\alpha_{n} u+\left(1-\alpha_{n}\right) F_{r_{n}} T_{r_{n}} x_{n},
$$

where $\left\{\alpha_{n}\right\} \subset[0,1]$ and $\left\{r_{n}\right\} \subset(0, \infty)$ are such that $\lim _{n \rightarrow \infty} \alpha_{n}=0, \sum_{n=0}^{\infty} \alpha_{n}=\infty$, $\sum_{n=0}^{\infty}\left|\alpha_{n+1}-\alpha_{n}\right|<\infty, \liminf _{n \rightarrow \infty} r_{n}>0$ and $\sum_{n=0}^{\infty}\left|r_{n+1}-r_{n}\right|<\infty$. The sequence $\left\{x_{n}\right\}$ converges strongly to $z \in \mathfrak{F}$, where $z=P_{\mathfrak{F}}(u)$.

In Theorem 3.1, $\gamma=1$ and $A, T_{1} \equiv I$, where $I$ is the identity mapping, then we have the following corollary.

Corollary 3.6 Let $H$ be a real Hilbert space and $T_{2}: C \rightarrow H$ be a continuous monotone mapping such that $\operatorname{VI}\left(C, T_{2}\right) \neq \emptyset$. Let $f$ be a contraction of $H$ into itself, and let $\left\{x_{n}\right\}$ be a sequence generated by $x_{1} \in H$ and

$$
x_{n+1}=\alpha_{n} f\left(x_{n}\right)+\left(1-\alpha_{n}\right) T_{r_{n}} x_{n},
$$

where $\left\{\alpha_{n}\right\} \subset[0,1]$ and $\left\{r_{n}\right\} \subset(0, \infty)$ are such that $\lim _{n \rightarrow \infty} \alpha_{n}=0, \sum_{n=0}^{\infty} \alpha_{n}=\infty$, $\sum_{n=0}^{\infty}\left|\alpha_{n+1}-\alpha_{n}\right|<\infty, \liminf _{n \rightarrow \infty} r_{n}>0$ and $\sum_{n=0}^{\infty}\left|r_{n+1}-r_{n}\right|<\infty$. The sequence $\left\{x_{n}\right\}$ converges strongly to $z \in F\left(T_{1}\right)$, where $z=P_{F\left(T_{1}\right)}(z)$.

Remark 3.7 Our results extend and unify most of the results that have been proved for these important classes of nonlinear operators. In particular, Theorem 3.1 extends Theorem 3.1 of Iiduka and Takahashi [12] and Zegeye et al. [25], Corollary 3.2 of Su et al. [29] in the sense that our convergence is for the more general class of continuous pseudocontractive and continuous monotone mappings. Corollary 3.4 also extends Theorem 4.2 
of Iiduka and Takahashi [12] in the sense that our convergence is for the more general class of continuous pseudo-contractive and continuous monotone mappings.

Competing interests

The authors declare that they have no competing interests.

Authors' contributions

All authors contributed equally and significantly in writing this paper. All authors read and approved the final manuscript.

\section{Author details}

${ }^{1}$ Department of Mathematics, Faculty of Science, Naresuan University, Phitsanulok, 65000, Thailand. ${ }^{2}$ Department of Mathematics, School of Science, University of Phayao, Phayao, 56000, Thailand.

\section{Acknowledgements}

The authors would like to thank Naresuan University and the university of Phayao. Moreover, the authors would like to thank the referees for reading this paper carefully, providing valuable suggestions and comments, and pointing out a major error in the original version of this paper.

Received: 29 May 2013 Accepted: 18 August 2013 Published: 5 September 2013

\section{References}

1. Borwein, FE: Nonlinear monotone operators and convex sets in Banach spaces. Bull. Am. Math. Soc. 71, 780-785 (1965)

2. Bruck, RE: On the weak convergence of an ergodic iteration for the solution of variational inequalities for monotone operators in Hilbert space. J. Math. Anal. Appl. 61, 159-164 (1977)

3. Noor, MA, Noor, Kl, Al-Said, E: Iterative methods for solving general quasi-variational inequalities. Optim. Lett. 4, 513-530 (2010)

4. Takahashi, W: Nonlinear complementarity problem and systems of convex inequalities. J. Optim. Theory Appl. 24, 493-508 (1978)

5. Yao, Y, Liou, YC, Yao, JC: An extragradient method for fixed point problems and variational inequality problems. J. Inequal. Appl. (2007). doi:10.1055/2007/38752

6. Yao, Y, Liou, YC, Yao, JC: An iterative algorithm for approximating convex minimization problem. Appl. Math. Comput. $188,648-656(2007)$

7. Yao, Y, Noor, MA, Liou, YC: Strong convergence of a modified extragradient method to the minimum-norm solution of variational inequalities. Abstr. Appl. Anal. (2012). doi:10.1155/2012/817436

8. Yao, Y, Cho, YJ, Chen, R: An iterative algorithm for solving fixed point problems, variational inequality problems and mixed equilibrium problems. Nonlinear Anal. 71, 3363-3373 (2009)

9. Zegeye, H, Shahzad, N: Strong convergence for monotone mappings and relatively weak nonexpansive mappings. Nonlinear Anal. 70, 2707-2716 (2009)

10. Borwein, JM: Fifty years of maximal monotonicity. Optim. Lett. 4, 473-490 (2010)

11. Browder, FE, Petryshyn, WV: Construction of fixed points of nonlinear mappings in Hilbert spaces. J. Math. Anal. Appl. 20, 197-228 (1967)

12. liduka, H, Takahashi, W, Toyoda, M: Approximation of solutions of variational inequalities for monotone mappings. Panam. Math. J. 14, 49-61 (2004)

13. Liu, F, Nashed, MZ: Regularization of nonlinear ill-posed variational inequalities and convergence rates. Set-Valued Anal. 6, 313-344 (1998)

14. Nakajo, K, Takahashi, W: Strong and weak convergence theorems by an improved splitting method. Commun. Appl. Nonlinear Anal. 9, 99-107 (2002)

15. Lin, LJ, Takahashi, W: A general iterative method for hierarchical variational inequality problems in Hilbert spaces and applications. Positivity (2012). doi:10.1007/s11117-012-0161-0

16. Halpern, B: Fixed points of nonexpansive maps. Bull. Am. Math. Soc. 73, 957-961 (1967)

17. Lions, PL: Approximation de points fixes de contractions. C. R. Acad. Sci. Paris Sér. A-B 284, 1357-1359 (1977)

18. Wittmann, R: Approximation of fixed points of nonexpansive mappings. Arch. Math. 58, 486-491 (1991)

19. Reich, S: Strong convergence theorems for resolvents of accretive operators in Banach spaces. J. Math. Anal. Appl. 75 287-292 (1980)

20. Shioji, N, Takahashi, W: Strong convergence of approximated sequences for nonexpansive mappings in Banach spaces. Proc. Am. Math. Soc. 70, 45-57 (2009)

21. Zegeye, H, Shahzad, N: Viscosity approximation methods for fixed-points problems. Appl. Math. Comput. 191 155-163 (2007)

22. Moudafi, A: Viscosity approximation methods for fixed-points problems. J. Math. Anal. Appl. 241, 46-55 (2000)

23. Zegeye, $\mathrm{H}$, Shahzad, N, Mekkonen, T: Viscosity approximation methods for pseudocontractive mappings in Banach spaces. Appl. Math. Comput. 185, 538-546 (2007)

24. Marino, G, Xu, HK: A general iterative method for nonexpansive mapping in Hilbert spaces. J. Math. Anal. Appl. 318, 43-52 (2006)

25. Zegeye, $\mathrm{H}$, Shahzad, N: Strong convergence of an iterative method for pseudo-contractive and monotone mappings. J. Glob. Optim. 54, 173-184 (2012). doi:10.10007/s10898-011-9755-5

26. Xu, HK: Iterative algorithms for nonlinear operators. J. Lond. Math. Soc. 66, 240-256 (2002)

27. Zegeye, $\mathrm{H}$ : An iterative approximation methods for a common fixed point of two pseudo-contractive mappings. ISRN Math. Anal. 2011, Article ID 621901 (2011). doi:10.5402/2011/621901 
28. Takahashi, W, Zembayashi, $\mathrm{K}$ : Strong and weak convergence theorems for equilibriums problems and relatively nonexpansive mappings in Banach spaces. Nonlinear Anal. 70, 45-57 (2009)

29. Su, Y, Shang, M, Qin, X: An iterative method of solution for equilibrium and optimization problems. Nonlinear Anal. $69,2709-2719(2008)$

doi:10.1186/1687-1812-2013-233

Cite this article as: Wangkeeree and Nammanee: New iterative methods for a common solution of fixed points for pseudo-contractive mappings and variational inequalities. Fixed Point Theory and Applications 2013 2013:233.

Submit your manuscript to a SpringerOpen ${ }^{\odot}$ journal and benefit from:

- Convenient online submission

- Rigorous peer review

- Immediate publication on acceptance

- Open access: articles freely available online

- High visibility within the field

- Retaining the copyright to your article

Submit your next manuscript at $\gg$ springeropen.com 